\title{
Evaluación del impacto de la cuarentena por la pandemia de COVID-19 en la transmisión perinatal del VIH en Buenos Aires, Argentina
}

\author{
Recibido: 29/7/21 Aceptado: 5/10/21
}

\begin{abstract}
Diego Cecchini, 2 , Jamile Ballivian²,3, Marcela Ortiz de Zárate ${ }^{3}$, María José Rolón ${ }^{4}$, Silvina Ivalo ${ }^{5}$, María Teresa Rodriguez Brieschke ${ }^{6}$, María Fernanda Consalvo7, Marina Martinez'; Florencia Verdi Brusati'; Claudia Rodriguez'; María Laura Samaniego ${ }^{2}$, Verónica Rossi ${ }^{4}$, Mónica González Alcántara5 , Silvia Perez Macri $^{5}$, Patricia Coll ${ }^{5}$, Irene Foradori ${ }^{6}$, Alejandro Hakim ${ }^{6}$, Damián Serrano ${ }^{6}$, Claudia Scalise ${ }^{6}$, Manuela Bulló ${ }^{6}$, Juliana Caccavo ${ }^{7}$, Mara de Bernardi ${ }^{7}$, Andrea Scardigno ${ }^{8}$, Constanza Johnston ${ }^{8}$, Silvina Vulcano ${ }^{3}$, Adriana Duran ${ }^{3}$.
\end{abstract}

${ }^{*}$ In memorian

\section{RESUMEN}

Objetivos: Describir variables epidemiológicas clave durante el año 2020 (pandemia de COVID-19) con respecto a la prevención de la transmisión perinatal (TP) del VIH en Ciudad de Buenos Aires (CABA), comparando con períodos previos.

Métodos: Análisis retrospectivo de los datos agregados de TP de las principales maternidades de CABA. El año pandémico (2020) se comparó con los años no pandémicos 2018 y 2019.

Resultados: Se observó una reducción del total de nacimientos en 2020 en comparación con 2019 y 2018 (11.640 vs. 14.031 y 15,978, respectivamente). La proporción de nacidos vivos en madres $\mathrm{VIH}+(\mathrm{MEV})$ fue $0,88 \%$ en 2020, sin diferencia con 2019 y $2018(0,94 \%$ y $0,93 \%)$, p> 0,05 para todas las comparaciones. Entre las MEV, el diagnóstico intraparto fue del 2,9\% para 2020 , sin diferencias con 2019 (2,25\%) y 2018 (9,3\%), p> 0,05 (todas las comparaciones); el 8,8\% comenzó el tratamiento antirretroviral con $>28$ semanas de edad gestacional en 2020 frente al 16\% y el 18,05\% en 2018 y 2019 ( $p>0,05$, todas las comparaciones). La prevalencia de la carga viral indetectable en el momento del parto fue del $67 \%$ en 2020 frente al $64 \%$ en 2018 y del $65,4 \%$ en 2019 ( $p>0,05$, todas las comparaciones). La transmisión perinatal fue $0 \%$ en 2020 vs. 1,33\% en 2018 y $2,25 \% 2019$ ( $p>0,05$, todas las comparaciones).

Conclusiones: En la primera ola de la pandemia de COVID-19 no se observaron cambios en la proporción de

\begin{abstract}
' Hospital General de Agudos Cosme Argerich, Almirante Brown 240, Ciudad Autónoma de Buenos Aires; Helios Salud, Perú 1511/15, Ciudad Autónoma de Buenos Aires. ${ }^{2}$ Helios Salud, Perú 1511/15, Ciudad Autónoma de Buenos Aires; Ministerio de Salud, Gobierno de la Ciudad Autónoma de Buenos Aires.

${ }^{3}$ Ministerio de Salud, Gobierno de la Ciudad Autónoma de Buenos Aires.

${ }^{4}$ Hospital Materno Infantil Ramón Sardá, Esteban de Luca 2151, Ciudad Autónoma de Buenos Aires.

${ }^{5}$ Hospital General de Agudos Juan A. Fernández, Av. Cerviño 3356, Ciudad Autónoma de Buenos Aires. ${ }^{6}$ Hospital General de Agudos José M. Ramos Mejía, Gral. Urquiza 609, Ciudad Autónoma de Buenos Aires. ${ }^{7}$ Hospital General de Agudos Donación Francisco Santojanni, Pilar 950, Ciudad Autónoma de Buenos Aires. ${ }^{8}$ Hospital General de Agudos Jose María Penna, Avenida Almafuerte 406, Ciudad Autónoma de Buenos Aires.
\end{abstract}

Autor para correspondencia: Dr. Diego Cecchini, Hospital Cosme Argerich, Almirante Brown 240, Buenos Aires 1155AHD, Argentina. Tel: +54 11 4121-0828. Fax: +54 11 4307-5952. E-mail: diegocec@gmail.com. Helios Salud SA. Perú 1511/15, Buenos Aires, C1141ACG, Argentina. Tel: +5411 43637400. E-mail: dcecchini@heliossalud.com.ar.

Los autores no presentan conflictos de intereses que declarar.

MEV asistidas, diagnóstico intraparto de $\mathrm{VIH}$, inicio tardío del TARV y TP en CABA.

Palabras clave: VIH, COVID-19, transmisión perinatal, América Latina, Argentina. 


\section{Introducción}

En enero de 2020, la Organización Mundial de la Salud (OMS) declaró el brote mundial de COVID-19 como una emergencia de salud pública, y posteriormente, tras comprometer los seis continentes, como pandemia a partir del 11 de marzo de 2020. El agente causal de COVID-19, el coronavirus del síndrome respiratorio agudo grave 2 (SARS-CoV-2), fue descripto a principios de 2020 a raíz del estudio del brote inicial en la provincia de Wuhan, China, a fines de 2019 (1-3).

El crecimiento exponencial en el número de casos en todos los continentes ha llevado a implementar estrategias para restringir el movimiento de la población (cuarentena masiva) con el objetivo de controlar la epidemia y evitar la saturación de los sistemas de atención de salud. Estas restricciones se han aplicado en numerosos países y se han desarrollado protocolos regionales que incluyen recomendaciones para el distanciamiento social, cierre de escuelas, restricciones a las reuniones sociales y en el transporte (a nivel jurisdiccional, nacional e internacional), y limitación de toda actividad considerada no esencial (4-5).

Argentina resultó ser uno de los países más afectados por la pandemia de COVID-19 a nivel mundial. Si bien el primer caso fue reportado el 3 de marzo de 2020, el Estado Nacional decretó el inicio del aislamiento social preventivo y obligatorio (ASPO) el 20 de marzo de ese año, extendiéndose durante 234 días. Si bien la epidemia presentó su epicentro inicialmente en el Área Metropolitana de Buenos Aires, que comprende la Ciudad Autónoma de Buenos Aires (CABA) y 40 municipios circundantes, posteriormente se expandió a todo el país (6-8).

Según datos reportados por Ministerio de Salud de Argentina, se estima que en el país viven 139.000 personas con $\mathrm{VIH}$, de las cuales solamente el $80 \%$ conoce su diagnóstico. La relación varón/mujer es de 2,5 a 1, siendo la principal vía de transmisión las relaciones sexuales sin uso de preservativo (98\%). De acuerdo a los datos del último boletín epidemiológico, la transmisión perinatal o vertical constituye el 1,9\% y 0,4\% del modo de transmisión en mujeres y hombres cis, respectivamente. El total de niños expuestos nacidos en 2017 estudiados fue de 1559, de los cuales 49 resultaron positivos para el VIH. Esto supone una tasa de transmisión perinatal de 3,1\% (tasa positivos/expuestos). Si se toma solo a los 1073 niños y niñas estudiados que concluyeron el proceso diagnóstico (casos cerrados), la tasa de transmisión perinatal es de $4,6 \%$. En 2018 , las tasas se ubicaron en $2,4 \%$, considerando el total de niños expuestos, y en $4,7 \%$ si solo se toman los casos cerrados (9).

Teniendo en cuenta datos de CABA, entre enero de 2003 y diciembre de 2019 se notificaron 3821 partos en mujeres con VIH (MEV) desde los hospitales públicos y un reducido número de partos de otras maternidades de los subsistemas de obra social y privada, siendo el principal distrito en volumen de atención de esta población. Del total de partos, 1579 (42,35\%) correspondieron a mujeres residentes de la ciudad; $2116(56,75 \%)$ a residentes del conurbano bonaerense (que comprende 24 municipios de la provincia de Buenos Aires circundantes a CABA) y $33(0,88 \%)$ a residentes de otras jurisdicciones. Para el período 2003-2017, el porcentaje de cobertura de tratamiento antirretroviral (TARV) prenatal fue mayor al $80 \%$ (2935), con un aumento progresivo hacia el final del período. CABA presenta un descenso sostenido de la transmisión vertical del VIH a partir de 2013 manteniéndose por debajo del $3,5 \%$ a partir de 2017 , sin alcanzar tasas menores al $2 \%(10)$.

En el marco del "Plan de acción para la prevención y el control de la infección por el VIH y las infecciones de transmisión sexual 2016-2021", Argentina tiene como objetivo programático lograr una tasa de transmisión perinatal del VIH del $2 \%$ o menos (11). Dicho objetivo aún no fue logrado ni en CABA ni a nivel país (9). En este contexto, la pandemia de COVID-19 y las medidas de restricción de la circulación implementadas profundizaron en nuestro medio las brechas sociales, desigualdades socioeconómicas y de género que, sumadas a factores estructurales como la fragmentación del sistema sanitario, pueden tener un impacto deletéreo en el acceso a la salud de las poblaciones más vulnerables, como las $\operatorname{MEV}(9,12)$. No se dispone de datos de América Latina en relación a los efectos de la pandemia de COVID-19 en la transmisión perinatal del VIH. Teniendo en cuenta esta situación, resulta de importancia describir, considerando la cuarentena por la pandemia de COVID-19, variables epidemiológicas inherentes a la transmisión perinatal del VIH en CABA a fin de realizar un diagnóstico de situación que permita guiar políticas de salud pública a nivel local y regional. 


\section{Materiales y métodos}

Análisis retrospectivo de datos agregados de las principales maternidades de CABA. Se incluyeron las siguientes instituciones: Hospital General de Agudos Cosme Argerich, Hospital Materno Infantil Ramón Sardá, Hospital General de Agudos Juan A. Fernández, Hospital General de Agudos José M. Ramos Mejía, Hospital General de Agudos Donación Francisco Santojanni y Hospital General de Agudos José María Penna. Dichos hospitales cubren aproximadamente el $70 \%$ de los partos en el distrito considerando diferentes áreas programáticas del mismo (detalle en Figura 1). El año pandémico (2020) se comparó con los años no pandémicos 2018 y 2019 individualmente, considerando los siguientes indicadores epidemiológicos clave:

- Número total de partos.

- Partos en MEV (nacidos vivos).

- Diagnóstico intraparto de VIH: test rápido positivo para VIH confirmado posteriormente por enzimoinmunoensayo y carga viral.

- Inicio tardío de TARV: inicio con $>28$ semanas de gestación.

- Carga viral al final de la gestación (semana 36 de edad gestacional).

- Transmisión perinatal del VIH: tasa recién nacidos positivos/recién nacidos expuestos.

Se consideró infectado a aquel recién nacido con al menos 2 PCR en ADN proviral positivas (muestra inicial positiva, confirmada por segunda muestra).

Se realizaron comparaciones usando el X2-test y el test de Fisher.

\section{Resultados}

Se observó una reducción del total de nacimientos en 2020 en comparación con 2019 y 2018 (11.640 vs. 14.031 y 15.978, respectivamente). La proporción de nacidos vivos en MEV fue $0,88 \%(n=103)$ en 2020 , sin diferencia con 2019 y $2018(0,94 \%, n=133 ; 0,93 \%, n=150), p>0,05$ para todas las comparaciones (Tabla 1).
Entre las MEV, el diagnóstico intraparto fue del 2,9\% ( $\mathrm{n}=$ 3) para 2020, sin diferencias entre $2019(2,25 \%, n=3)$ y $2018(9,3 \%, n=14), p>0,05$ (todas las comparaciones). El 8,8\% comenzó el TARV tardíamente en 2020 frente al $16 \%$ y el $18,05 \%$ en 2018 y 2019 ( $p>0,05$, todas las comparaciones).

La prevalencia de la carga viral indetectable en el momento del parto fue del $67 \%$ en 2020 frente al $64 \%$ en 2018 y del $65,4 \%$ en 2019 ( $p>0,05$, todas las comparaciones).

La transmisión perinatal fue $0 \%$ en 2020 vs. $1,33 \%$ en 2018 y $2,25 \% 2019$ ( $p>0,05$, todas las comparaciones). El número total de casos de recién nacidos infectados por hospital por año se presentan en la Tabla 1.

Figura 1. Distribución histórica de partos (porcentaje) en embarazadas viviendo con VIH en los hospitales públicos de la Ciudad Autónoma de Buenos Aires (2003-2019) participantes en la evaluación del impacto de la pandemia COVID-19 en la transmisión perinatal.

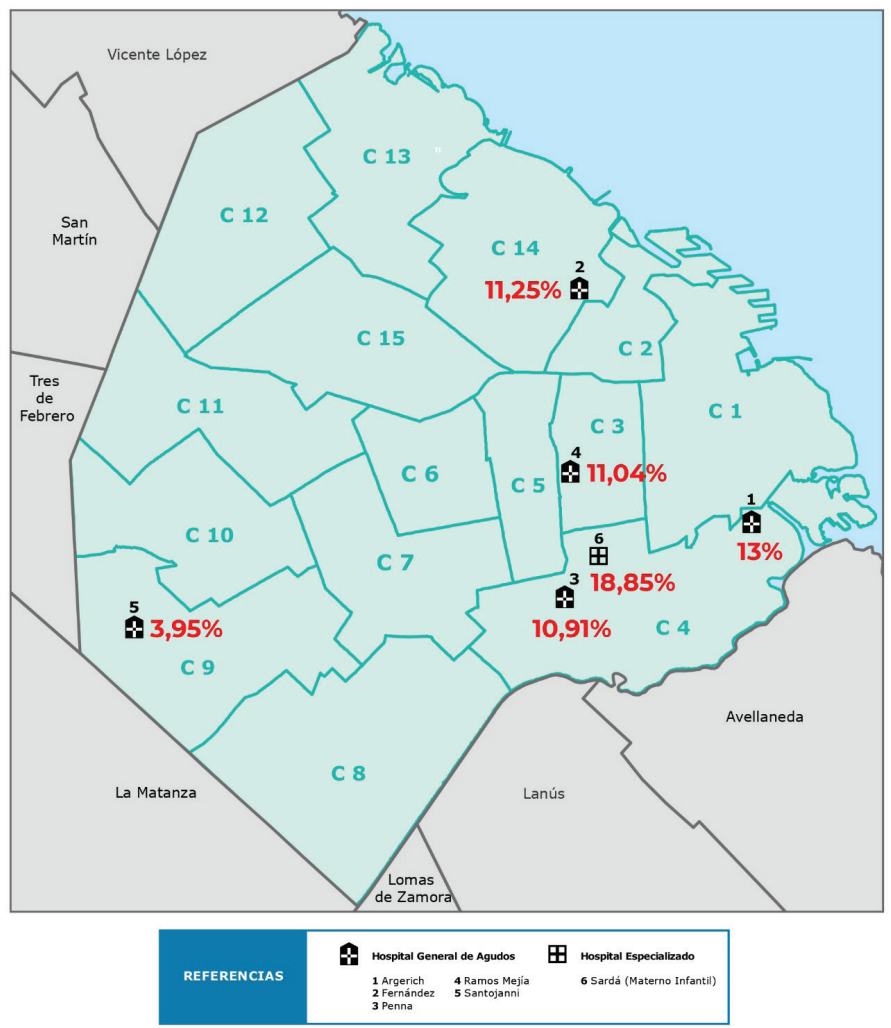

Fuente: Ministerio de Salud, Ciudad de Buenos Aires. Gráfico adaptado de la Dirección de Estadísticas y Censos https://www. estadisticaciudad.gob.ar/eyc/wp-content/uploads/2021/03/ SA072101.pdf (acceso el 4 de mayo de 2021) 
Tabla 1. Total de nacimientos, partos en mujeres infectadas por VIH y casos de transmisión perinatal en las principales maternidades de la Ciudad de Buenos Aires (período 2018-2020).

\begin{tabular}{|c|c|c|c|c|}
\hline Hospital & Año & $\begin{array}{c}\text { Total de } \\
\text { nacimientos } \\
\text { (n) }\end{array}$ & $\begin{array}{l}\text { Mujeres } \\
\text { VIH+ que } \\
\text { parieron } \\
\text { recién } \\
\text { nacidos } \\
\text { vivos }(n)\end{array}$ & $\begin{array}{c}\text { Transmisión } \\
\text { perinatal, } \\
\text { casos }(n)\end{array}$ \\
\hline \multirow[t]{3}{*}{ Argerich } & 2018 & 1740 & 31 & 1 \\
\hline & 2019 & 1646 & 22 & 0 \\
\hline & 2020 & 1149 & 15 & 0 \\
\hline \multirow[t]{3}{*}{ Sardá } & 2018 & 5750 & 51 & 1 \\
\hline & 2019 & 5077 & 44 & 0 \\
\hline & 2020 & 4831 & 39 & 0 \\
\hline \multirow[t]{3}{*}{ Fernández } & 2018 & 1501 & 33 & 0 \\
\hline & 2019 & 1502 & 26 & 0 \\
\hline & 2020 & 1030 & 19 & 0 \\
\hline \multirow[t]{3}{*}{ Santojanni } & 2018 & 2357 & 9 & 0 \\
\hline & 2019 & 2108 & 11 & 0 \\
\hline & 2020 & 2010 & 13 & 0 \\
\hline \multirow[t]{3}{*}{ R. Mejía } & 2018 & 1313 & 14 & 0 \\
\hline & 2019 & 887 & 11 & 2 \\
\hline & 2020 & 663 & 5 & 0 \\
\hline \multirow[t]{3}{*}{ Penna } & 2018 & 3317 & 12 & 0 \\
\hline & 2019 & 2811 & 19 & 1 \\
\hline & 2020 & 1957 & 12 & 0 \\
\hline
\end{tabular}

\section{Discusión}

La pandemia de COVID-19 ha impactado drásticamente en la salud pública a nivel mundial, representando asimismo un desafío sin precedentes para el sistema sanitario de los países de América Latina, incluyendo Argentina (13). Por tal motivo, las estrategias de cuarentena han sido de fundamental importancia para reducir en el corto-mediano plazo la incidencia de casos y evitar la saturación del sistema sanitario (14-15). Sin embargo, estas restricciones tienen impacto deletéreo a nivel económico, psicosocial y en el acceso al sistema de salud para el diagnóstico y tratamiento de patologías agudas y crónicas (16-18). En este sentido, las poblaciones vulnerables al estigma y la discriminación, como las personas que viven con $\mathrm{VIH}$, pueden ser particularmente perjudicadas por las estrategias de cuarentena tanto por el impacto psicosocial de la misma como por dificultades en el acceso a los controles médicos y al retiro de medicación. Asimismo, la pandemia puede impactar negativamente en el diagnóstico de $\mathrm{VIH}$, restringiendo el acceso a los centros de testeo (19-22).

Un análisis de ONUSIDA encontró un impacto negativo de la pandemia de COVID-19 en el acceso al diagnóstico y tratamiento del $\mathrm{VIH}$ en mujeres embarazadas en determinados países, particularmente en etapas iniciales de la pandemia (abril 2020). Estas disrupciones en los servicios de atención perinatal fueron revirtiéndose en la mayoría de los casos para junio del mismo año (23). Por otra parte, datos del Fondo Global contra HIV, Tuberculosis y Malaria muestran efectos negativos de la pandemia en servicios de atención materno-infantil en países de África y Asia, con descensos del 5 al $66 \%$ en el número de primeras visitas prenatales. Las causas de estas disrupciones en la atención fueron múltiples e incluyeron tanto factores inherentes al individuo y la comunidad como aquellos propios de las instituciones de salud. Las primeras incluyeron: temor a adquirir infección por SARS-CoV-2 al concurrir a centros de salud, dificultad para acceder al transporte, acatamiento de la prohibición de circulación ordenada por las autoridades sanitarias, decisión de retrasar consultas médicas, entre otras. En cuanto a las propias del sistema de salud: reducción o suspensión en la atención en determinados servicios/instituciones, afectación de instituciones exclusivamente a la atención de casos de COVID-19, reducción de las campañas de salud promoviendo consultas médicas no vinculadas a COVID-19, reducción en horarios de atención, cierre de centros de salud, falta de comunicación a la comunidad sobre la reactivación de servicios o prestaciones previamente suspendidos, etc... De esta forma, la pandemia pone en riesgo la continuidad de programas contra HIV, tuberculosis y malaria a nivel mundial $(24,25)$.

Ante la falta de datos locales, y teniendo en cuenta que tanto a nivel país como nivel de la CABA aún no ha sido logrado el objetivo de transmisión perinatal del $\mathrm{VIH}<2 \%(9-10)$, resulta de crucial importancia evaluar si este indicador no pudo haber empeorado producto de la pandemia de COVID-19 y las medidas de restricción de la circulación impuestas como política sanitaria. Esto es particularmente importante teniendo en cuenta tres factores: 1) que el Área Metropolitana de Buenos Aires fue una de las áreas más afectadas por la pandemia a nivel país; 2) que dicha área concentra la mayor cantidad de personas que viven con VIH y de partos en mujeres 
con VIH y 3) que la cuarentena (ASPO) en esta área fue una de las más extensas del mundo (6-10).

Por tal motivo, hemos adoptado la estrategia de analizar datos agregados de las principales maternidades de CABA en los últimos tres años (2018 y 2019, años no pandémicos, y 2020, año pandémico) a fin de detectar rápidamente deterioros en los indicadores inherentes a la evaluación de la transmisión perinatal del VIH y de, eventualmente, promover la implementación expeditiva de estrategias de mitigación.

Este estudio muestra posiblemente un impacto objetivo de la cuarentena en la población global de mujeres embarazadas: el número de partos en 2020 en CABA fue inferior a los dos años previos (no pandémicos). Esto puede observarse tanto considerando los datos globales como desagregados por institución (Tabla 1). Teniendo en cuenta que una importante proporción de mujeres residentes en localidades aledañas de la provincia de Buenos Aires (conurbano bonaerense) pare en CABA, es posible que el descenso en partos totales sea una expresión de la imposibilidad de dichas mujeres de trasladarse a este distrito para los controles obstétricos y el parto. Esta hipótesis deberá confirmarse con futuros análisis epidemiológicos por parte de las autoridades sanitarias de las jurisdicciones intervinientes.

Los resultados de la presente investigación no muestran, comparando $2018-2019$ vs. 2020 , deterioro en ningún indicador propio de la atención de la MEV: partos en MEV, proporción de MEV con diagnóstico intraparto, inicio tardío de TARV, carga viral no detectable cercana a la finalización del embarazo y tasa de transmisión vertical. Teniendo en cuenta el contexto sanitario a priori desfavorable, estos resultados son alentadores en términos de la posibilidad de lograr el objetivo de transmisión perinatal $<2 \%$ al cual CABA viene aproximándose en los últimos años (10).

Es de destacar que la tasa de transmisión perinatal, considerando datos agrupados de los hospitales intervinientes (que son altamente representativos de la situación de CABA) fue de cero en 2020. Considerando que el escenario de cuarentena en contexto de pandemia de COVID-19 es desfavorable, este resultado podría atribuirse a diferentes factores vinculables a estrategias de mitigación adoptadas por las instituciones intervinientes para garantizar la atención de las MEV. Dichas estrategias pueden ser variables en función de cada hospital, pero incluyen conceptualmente el desarrollo empírico o fortalecimiento de circuitos donde la atención de la MEV es prioritaria: mantenimiento de la atención y prácticas obstétricas ambulatorias y de internación; priorización de la atención programada y no programada de las MEV en los servicios de infectología y obstetricia; habilitación de consultas por telemedicina; dispensación de tratamiento antirretroviral por más de un mes; grupos de trabajo interdisciplinarios específicos para la atención de $\operatorname{MEV~(26);~fortalecimiento~del~}$ seguimiento de las MEV a través de los servicios sociales y de promoción y protección de la salud; fortalecimiento de la comunicación con los centros de atención primaria del área programática y facilitación de permisos de circulación a las pacientes, entre otros. Es de destacar que la facilitación de permisos de circulación para personas con VIH/SIDA, cuyo modelo fue gestionado por la Dirección de Respuesta al VIH, ITS, Hepatitis Virales y Tuberculosis, pudo haber colaborado con el traslado a CABA de MEV de otras jurisdicciones. Asimismo, la mayor disponibilidad de TARV basado en inhibidores de la integrasa en Argentina en 2020, que ha demostrado ser superior a otras drogas en la velocidad en que logra la supresión viral y la prevalencia de carga viral no detectable al final de la gestación, pudo ser otro factor relevante en la prevención de la transmisión vertical del VIH (27-28). Esta hipótesis deberá comprobarse en futuras investigaciones, no pudiéndose en este estudio hacerse conclusiones al respecto.

Nuestro estudio presenta determinadas limitaciones que deben mencionarse a fin de permitir una mejor interpretación de los resultados obtenidos. En primer lugar, el número de instituciones participantes, si bien altamente representativa, es limitada y solamente perteneciente al sistema público de salud. Teniendo en cuenta que el impacto de la pandemia y la cuarentena comprometen a todo el sistema sanitario, resultaría de importancia evaluar si hubo empeoramiento de estos indicadores en el sector privado de salud (medicina prepaga y obras sociales) en este distrito. En segundo lugar, para la comparación de 2020 (año pandémico) con 2018 y 2019 (no pandémicos), 2020 fue tomado en su totalidad a los fines de facilitar un análisis global de datos, ya que una comparación mes por mes o del período estricto de ASPO hubiera sido dificultosa y compleja en términos de recolección y consolidación de datos de cada centro. No obstante, el ASPO (en sus fases más restrictivas) correspondió al $65 \%$ de los días calendario 2020. En tercer lugar, nuestro estudio no contempló qué 
estrategias de TARV utilizó cada institución en cada año, impidiendo detectar cambios en la tendencia de prescripción y en qué medida se indicó tratamiento con inhibidores de integrasa a las MEV. El impacto de las diferentes pautas de prescripción de TARV en las tasas de transmisión perinatal deberá ser objeto de futuros estudios. En cuarto lugar, nuestro análisis no incluye la jurisdicción de origen de las MEV, pero podemos plantear la hipótesis de que estas pacientes, utilizando los permisos de circulación oficiales para personas que viven con $\mathrm{VIH}$, pudieron trasladarse para su control prenatal y parto en CABA desde otras localidades, lo que deberá ser corroborado o refutado por las autoridades sanitarias jurisdiccionales. Por último, la evaluación de la transmisión perinatal del VIH se hizo en base al indicador "tasa recién nacidos positivos/ recién nacidos expuestos". Dicho indicador tiende a subestimar la transmisión perinatal al no considerar los casos cerrados, es decir con estudios y seguimiento neonatal completos (indicador "tasa positivos/casos cerrados"). No obstante, un indicador basado en "casos cerrados" no hubiera permitido obtener rápidamente la información aquí presentada, ya que hubiera requerido un seguimiento completo de cada niño expuesto (incluyendo el enzimoinmunoensayo a partir del año de vida). Teniendo en cuenta esto, los datos presentados (particularmente para el año 2020) deben considerarse como preliminares. Sin embargo, podrían ser suficientes para demostrar que no hubo ascenso en la transmisión perinatal del VIH en CABA en el contexto de la "primera ola" de la pandemia de COVID-19.

Teniendo en cuenta la dinámica de esta pandemia con posibilidad de nuevas olas, desarrollo de variantes con perfil patogénico diferencial, sumados a las condiciones de pobreza estructural y dificultad en el acceso al testeo y vacunación de COVID-19 que presentan diferentes regiones de América Latina (29-32), es de esperar un impacto de la misma en otras patologías, particularmente aquellas de carácter crónico. Teniendo en cuenta que la tasa de transmisión perinatal del VIH es un indicador sumamente sensible en el desarrollo de los programas de $\mathrm{VIH} / \mathrm{SIDA}$ a nivel local y regional, nuestro estudio aporta información inicial de un tema que requiere futuras investigaciones en las Américas.

\section{Agradecimientos}

A Luciano Fresia, de Helios Salud, por su colaboración en la adaptación de la figura.

\section{Contribución de los autores}

DMC, JB, MOZ, MJR, SI, MTRB, MFC, MM, MLS, SV, $A D$ contribuyeron al diseño, análisis final de datos, interpretación y redacción de primeros borradores del artículo. CR, FV, VR, MGA, SPM, PC, IF, AH, DS, CS, MB, JC, $\mathrm{MB}, \mathrm{AS}, \mathrm{CJ}$, fueron responsables de extracción de datos, análisis y consolidación de datos en cada institución participante. Todos los autores revisaron y aprobaron la versión final del manuscrito. 


\section{Bibliografía}

1. Guan W-J, Ni Z-Y, Hu Y, Liang W-H, Ou C-Q, He J-X, et al. Clinical Characteristics of Coronavirus Disease 2019 in China. N Engl J Med. 2020;382:1708-20.

2. Li Q, Guan X, Wu P, Wang X, Zhou L, Tong Y, et al. Early Transmission Dynamics in Wuhan, China, of Novel Coronavirus-Infected Pneumonia. N Engl J Med. 2020;382:1199-207.

3. Ludwig S, Zarbock A. Coronaviruses and SARS-CoV-2: A Brief Overview. Anesth Analg. 2020;131:93-6.

4. Anderson RM, Heesterbeek $H$, Klinkenberg $D$, Hollingsworth TD. How will country based mitigation measures influence the course of the COVID-19 epidemic? Lancet. 2020;395:931-934

5. Brooks SK, Webster RK, Smith LE, Woodland L, Wessely S, Greenberg N, ET AL. The psychological impact of quarantine and how to reduce it: rapid review of the evidence. Lancet. 2020;395:912-920.

6. Boletín Oficial de la República Argentina. AISLAMIENTOSOCIALPREVENTIVOYOBLIGATORIO. 2020. Disponible en https://www.boletinoficial.gob. ar/detalleAviso/primera/227042/20200320. Acceso el 6 de mayo de 2020.

7. Rearte A, Baldani A, Barcena Barbeira P, Domínguez $C$, Laurora $M$, Pesce $M$, et al. Características epidemiológicas de los primeros 116974 casos de Covid-19 en Argentina, 2020. Rev Argentina Salud Pública. 2020;1-9.

8. Leveau CM. Difusión espacio-temporal de muertes por COVID-19 en Argentina. Rev Panam Salud Publica. 2021;45:e3.

9. Ministerio de Salud. Respuesta al VIH y las ITS en Argentina. Año XXII. Diciembre 2020. Disponible en https://bancos.salud.gob.ar/recurso/boletin-sobreel-vih-sida-e-its-en-la-argentina-ndeg-37 Acceso el 6 de mayo de 2021

10. Ministerio de Salud,Ciudad de Buenos Aires. Situación Epidemiológica del VIH en la ciudad de Buenos Aires Mayo 2021. Disponible en https://www.buenosaires. gob.ar/salud/coordinaci\%C3\%B3n-salud-sexual-vihinfecciones-de-transmisi\%C3\%B3n-sexual/noticias/ situacion Acceso 15 de julio de 2021

11. Organización Panamericana de la Salud. Plan de acción para la prevención y el control de la infección por el VIH y las infecciones de transmisión sexual 2016-2021. Disponible en: https://iris.paho.org/ bitstream/handle/10665.2/34079/DC552017-spa. pdf? sequence $=1$ \&isAllowed $=y$ Acceso el 6 de mayo de 2021.
12. Naciones Unidas Argentina. COVID-19 en Argentina: impacto socioeconómico y ambiental. Disponible en: https://www.argentina.gob.ar/sites/default/ files/informecovid19_argentina.pdf Acceso el 6 de mayo de 2021.

13. The Lancet. COVID-19 in Latin America: a humanitarian crisis. Lancet. 2020;396:1463.

14. Garcia PJ, Alarcón A, Bayer A, Buss P, Guerra G, Ribeiro H, et al. COVID-19 Response in Latin America. Am J Trop Med Hyg. 2020;103:1765-1772.

15. Andrus JK, Evans-Gilbert T, Santos JI, Guzman MG, Rosenthal PJ, Toscano $C$, et al. Perspectives on Battling COVID-19 in Countries of Latin America and the Caribbean. Am J Trop Med Hyg. 2020;103:593-596.

16. Saqib MAN, Siddiqui S, Qasim M, Jamil MA, Rafique I, Awan UA, et al. Effect of COVID-19 lockdown on patients with chronic diseases. Diabetes Metab Syndr. 2020;14:1621-1623.

17. Arredondo A. The COVID-19 pandemic substantially complicates the usual challenges for patients with diabetes in Latin America. Diabetes Res Clin Pract. 2020;168:108394.

18. Araujo SEA, Leal A, Centrone AFY, Teich VD, Malheiro DT, Cypriano AS, et al. Impact of COVID-19 pandemic on care of oncological patients: experience of a cancer center in a Latin American pandemic epicenter. Einstein (Sao Paulo). 2020;19:eA06282.

19. Shiau S, Krause KD, Valera P, Swaminathan S, Halkitis PN. The Burden of COVID-19 in People Living with HIV: A Syndemic Perspective. AIDS Behav. 2020;24:22442249.

20. Lesko CR, Bengtson AM. HIV and COVID-19: Intersecting Epidemics With Many Unknowns. Am J Epidemiol. 2021;190:10-16.

21. Pinto RM, Park S. COVID-19 Pandemic Disrupts HIV Continuum of Care and Prevention: Implications for Research and Practice Concerning CommunityBased Organizations and Frontline Providers. AIDS Behav. 2020;24:2486-2489.

22. Ballivian J, Alcaide ML, Cecchini D, Jones DL, Abbamonte JM, Cassetti I, et al. Impact of COVID-19related stress and lockdown on mental health among people living with HIV in Argentina. J Acquir Immune Defic Syndr. 2020;85:475-482.

23. UNAIDS. COVID-19's impact on HIV vertical transmission services reversed. Disponible en: https://www.unaids.org/en/resources/presscentre/ featurestories/2020/october/20201027_covid19impact-hiv-vertical-transmission Acceso el 6 de mayo de 2021 
24. The Global Fund. The impact of COVID 19 on HIV, TB and Malaria and systems for health: a snapshot from 502 health facilities across Africa and Asia. Disponible en: https://www.theglobalfund.org/en/ updates/other-updates/2021-04-13-the-impactof-covid-19-on-hiv-tb-and-malaria-services-andsystems-for-health/ Acceso el 6 de mayo de 2021

25. Hogan $A B$, Jewell BL, Sherrard-Smith $E$, Vesga JF, Watson OJ, Whittaker $C$, et al. Potential impact of the COVID-19 pandemic on HIV, tuberculosis, and malaria in low-income and middle-income countries: a modelling study. Lancet Glob Health. 2021;9:e23

26. Cecchini D, Martinez M, Astarita V, Nieto C, Giesolauro $\mathrm{R}$, Rodriguez $\mathrm{C}$. Prevención de la transmisión vertical del VIH-1 en un hospital público de complejidad terciaria de Buenos Aires, Argentina. Rev Panam Salud Publica. 2011;30:189-95.

27. Kintu K, Malaba TR, Nakibuka J, Papamichael $C$, Colbers A, Byrne $K$, et al.. Dolutegravir versus efavirenz in women starting HIV therapy in late pregnancy (DolPHIN-2): an open-label, randomised controlled trial. Lancet HIV. 2020;7:e332-e339.

28. João EC, Morrison RL, Shapiro DE, Chakhtoura $N$, Gouvèa MIS, de Lourdes B Teixeira $M$, et al. Raltegravir versus efavirenz in antiretroviral-naive pregnant women living with HIV (NICHD P1081): an open-label, randomised, controlled, phase 4 trial. Lancet HIV. 2020;7:e322-e331.

29. Miller MJ, Loaiza JR, Takyar A, Gilman RH. COVID-19 in Latin America: Novel transmission dynamics for a global pandemic?. PLoS Negl Trop Dis. 2020;14:e0008265.

30. Cimerman S, Chebabo A, Cunha CAD, RodríguezMorales AJ. Deep impact of COVID-19 in the healthcare of Latin America: the case of Brazil. Braz J Infect Dis. 2020;24:93-95.

31. Rodriguez-Morales A.J., Sánchez-Duque J.A., Hernández-Botero S. Preparación y control de la enfermedad por coronavirus 2019 (COVID-19) en América Latina. Acta Med Peruana. 2020;37:3-7

32. Torres AG. Vacunas contra el SARS-CoV-2: ¿son una realidad para América Latina? Biomédica. 2020;40:424-6 
Assessment of the impact of the COVID-19 pandemic lockdown on perinatal HIV transmission in Buenos Aires, Argentina

Background: To describe key epidemiological variables in 2020 (COVID-19 pandemic) regarding prevention of mother-to-child transmission (MTCT) in Buenos Aires city (CABA) in comparison with previous periods.

Methods: Retrospective analysis of aggregated MTCT data was gathered from six principal maternity hospitals in Buenos Aires city. Pandemic year (2020) was compared to non-pandemic years 2018-19 individually considering key epidemiological variables.

Results: A reduction of total births was observed in 2020 compared to 2019 and 2018 (11640 vs. 14031 and 15978, respectively). Proportion of live births in HIV-infected women (HPW) was $0.88 \%$ in 2020 without difference with 2019 and 2018 (0.94\% and $0.93 \%)$, p> 0.05 for all comparisons. Among HPW, intrapartum diagnosis was $2.9 \%$ for 2020 , with no difference between 2019 (2.25\%) and 2018 (9.3\%), p $>0.05$ (all comparisons); $8.8 \%$ had antiretroviral therapy (ART) started $>28$ weeks of gestational age in 2020 vs. $16 \%$ and $18.05 \%$ in 2018 and 2019 ( $p>0.05$, all comparisons). Prevalence of undetectable viral load at delivery was $67 \%$ in 2020 vs $64 \%$ in 2018 and $65.4 \%$ in 2019 ( $p>0.05$, all comparisons). Perinatal transmission was $0 \%$ in 2020 vs $1.33 \%$ in 2018 and $2.25 \% 2019$ ( $p>0.05$, all comparisons)

Conclusions: In first wave of COVID 19 pandemic no changes in the proportion of HPW assisted, HIV intrapartum diagnosis, late ART initiation and MTCT-rate was observed in CABA.

Keywords: HIV; COVID-19; perinatal transmission; Latin America; Argentina. 